\title{
LATTICE SUMS OF HOMOGENEOUS FUNCTIONS
}

\author{
ROBERT S. CAHN
}

Abstract. An asymptotic estimate for a general lattice sum is derived using Fourier analysis techniques.

1. Let $x_{1}, \cdots, x_{n}$ be a basis of $R^{n}, n \geqq 2$, and $L=Z x_{1}+\cdots+Z x_{n}$ the lattice they generate. Let $B(T)=\{x \mid\|x\| \leqq T\}$ and $L(T)=\sum_{L \cap B(T)} 1$. Then it is known that $L(T)=\left(c_{n} / d\right) T^{n}+O\left(T^{n(n-1) /(n+1)}\right)$ where $c_{n}=$ Volume of $B(1)$ and $d=\left|\operatorname{Det}\left(x_{1}, \cdots, x_{n}\right)\right|$. We wish to prove an analogous estimate for a general homogeneous function.

2. Let $f(x)$ be a real-valued homogeneous function on $\boldsymbol{R}^{n}$, i.e., $f(r x)=$ $r^{\alpha} f(x)$ for all $r \geqq 0$ with the restriction that $\alpha \geqq 0$. We then define $c_{f}=$ $\int_{B(1)} f(x) d x$ and $L_{f}(T)=\sum_{x \in L \cap B(T)} f(x)$. We will prove

THEOREM. If $f$ is homogeneous and $f \in C^{[n / 2]+1}\left(R^{n}\right)$ then $L_{f}(T)=$ $\left(c_{f} / d\right) T^{n+\alpha}+O\left(T^{n(n-1) /(n+1)+\alpha}\right)$.

3. The Theorem may be obtained by the methods used in [1]. The only thing to prove is that the Fourier transform of $f \cdot X_{B(1)}(x)$ is $O\left(\|x\|^{-(n+1) / 2}\right)$ where $X_{B(1)}(x)$ is the characteristic function of $B(1)$ :

$$
\widehat{f \cdot X_{B(1)}}(x)=\int_{B(1)} e^{2 \pi i(y, x)} f(y) d y=\frac{1}{2 \pi i\|x\|} \int_{\partial B(1)} e^{2 \pi i(y, x)} g_{1}(y, x) d y
$$

with $g_{1}(y, x)([n / 2])$-times differentiable in $y$ (see the Lemma in [1] and Lemma 3 in [3]). By applying a partition of unity to $\partial B(1)$ and integrating by parts as in [2] our estimate follows. The proof of the Theorem is now carried out exactly as in [1] using the Poisson summation formula.

\section{BIBLIOGRAPHY}

1. R. Cahn, Lattice points and Lie groups. II, Trans. Amer. Math. Soc. (to appear).

2. W. Littman, Fourier transforms of surface-carried measures and differentiability of surface averages, Bull. Amer. Math. Soc. 69 (1963), 766-770. MR 27 \#5086.

3. B. Randol, A lattice-point problem, Trans. Amer. Math. Soc. 121 (1966), 257-268. MR 34 \#1291.

Department of Mathematics, University of Miami, Coral Gables, Florida 33124

Received by the editors October 13, 1972 .

AMS (MOS) subject classifications (1970). Primary $10 \mathrm{~J} 25$.

Key words and phrases. Lattice point, homogeneous function.

(c) American Mathematical Society 1973 